\title{
IMMERSIONS AND SURGERIES OF TOPOLOGICAL MANIFOLDS
}

\author{
BY J. ALEXANDER LEES ${ }^{1}$
}

Communicated by William Browder, December 9, 1968

In this announcement, we outline a version of Haefliger and Poenaru's Immersion Theorem [2] for topological manifolds. We then use our theorem to do surgery on topological manifolds, and obtain results such as the following: Let $M^{n}$ be a closed, almost parallelizable topological manifold (that is, the tangent bundle of $M-p$ is trivial, $p \in M$ ) which has the homotopy type of a finite complex. Then by a sequence of surgeries, $M$ can be reduced to an $[n / 2-1]$ connected almost parallelizable manifold.

In order to state the Immersion Theorem we give the following definitions: Let $M, M^{\prime}$ and $Q$ be topological manifolds, $M$ a compact locally flat submanifold of the open manifold $M^{\prime}$, with $\operatorname{dim} M^{\prime}$ $=\operatorname{dim} Q$.

Write $\operatorname{Im}_{M^{\prime}}(M, Q)$ for the semisimplicial complex of $M^{\prime}$ immersions of $M$ in $Q$; a simplex of $\operatorname{Im}_{M},(M, Q)$ is an immersion $f: \Delta \times U \rightarrow \Delta \times Q$ commuting with the projections on the standard simplex $\Delta$, where $U$ is a neighborhood of $M$ in $M^{\prime}$. Two such are identified if they agree on $\Delta \times\left(\right.$ a neighborhood of $M$ in $\left.M^{\prime}\right)$.

Write $R\left(T M^{\prime} \mid M, T Q\right)$ for the semisimplicial complex of representation germs of the tangent bundle of $M^{\prime}$ restricted to $M$ in the tangent bundle of $Q$; a simplex of $R\left(T M^{\prime} \mid M, T Q\right)$ is a microbundle map $\Phi$ of $\Delta \times T U$ in $\Delta \times T Q$ which commutes with projections on $\Delta, U$ a neighborhood of $M$ in $M^{\prime}$, such that the map of $\Delta \times T U$ in $\Delta \times U \times Q$ given by $\left(t, u, u^{\prime}\right) \rightarrow\left(t, u, \pi \Phi\left(t, u, u^{\prime}\right)\right)$ is an immersion on a neighborhood of $\Delta \times($ the diagonal of $M)$. Two such representations define the same representation germ if they agree on a neighborhood of $\Delta \times$ (the diagonal of $M$.

Observe that if $f$ is a simplex of $\operatorname{Im}_{M}(M, Q)$, the map $d f$ defined as follows, is a simplex of $R\left(T M^{\prime} \mid M, T Q\right): d f\left(t, u, u^{\prime}\right)=\left(t, f_{t} u, f_{t} u^{\prime}\right)$ where $u, u^{\prime} \in U, f(t, u)=\left(t, f_{t} u\right)$. We now state the Immersion Theorem. Suppose $M$ has a handlebody decomposition with all handles of index $<\operatorname{dim} Q$. Then the map $d: \operatorname{Im}_{M^{\prime}}(M, Q) \rightarrow R\left(T M^{\prime} \mid M, T Q\right)$ is a homotopy equivalence. $R$. Lashof has shown [6] that the hypothesis

1 The author was partially supported by NSF grant GP-8971. 
that $M$ has a handlebody decomposition can be removed, in case $\operatorname{dim} M<\operatorname{dim} M^{\prime}$.

We first prove the following

NEIGHBORHOOD $n$-ISOTOPY EXTENSION THEOREM. ${ }^{2}$ Let $E$, be euclidean $q$-space, $X$ a closed subset of $E^{q}, U \subset V$ neighborhoods of $X$ in $E^{q}$. Let $F: U \times I^{n} \rightarrow V^{a} \times I^{n}$ be an $n$-isotopy, that is, an embedding which commutes with projections on $I^{n}$. Then there is an ambient $n$-isotopy $H: V^{a} \times I^{n} \rightarrow V^{a} \times I^{n}$ fixed outside a compact set with $H\left(F_{0} x, t\right)=F(x, t)$ for $x \in U^{\prime}$, an open neighborhood of $X$ contained in $U$.

Proof. For clarity, we give the argument for $n=1$. Applying the lemma below starting at any level $s \in I$ in either direction we obtain $h_{8}$ and $U_{8}$ so that (replacing $F_{0}$ by $F_{8}$ ) the theorem holds for $x \in U$. and $t$ in an interval about $s$. We then use the compactness of the unit interval to construct the required isotopy $H$. Using an induction, this argument is generalized to prove the Neighborhood $n$-Isotopy Extension Theorem.

Lemma. There is an $\epsilon>0$ and a level preserving homeomorphism $h_{0}: V \times[0, \epsilon] \rightarrow V \times[0, \epsilon]$ fixed outside a compact set with $h_{0}\left(F_{0} x, t\right)$ $=F(x, t)$ for $x \in U_{0}, 0 \leqq t \leqq \epsilon, U_{0}$ an open neighborhood of $X$ contained in $U$.

Proof of the Lemma. Using the arguments in [4] we obtain the following

Sublemma. Let $h: \Delta^{i} \times E^{q-i} \rightarrow \Delta^{i} \times E^{q-i}$ be a homeomorphism which is the identity on a neighborhood of $\partial \Delta^{i} \times E^{q-i}$. Then if $h$ is close to the identity, there is a homeomorphism $g: \Delta^{i} \times E^{q-i} \rightarrow \Delta^{i} \times E^{q-i}$ which is the identity on a neighborhood of $\partial \Delta^{i} \times E^{q-i}$ and outside a compact set such that $g=h$ on a neighborhood of $\Delta^{i} \times 0$. Further, the map $h \rightarrow g$ is continuous in the compact-open topology.

Now let $K$ be a finite complex, $K^{i}$ the $i$-skeleton of $K$, with $U \supset K$ $\supset X$. We will construct a sequence of level preserving homeomorphisms $k^{i}$ such that $k^{i} F(x, t)=\left(F_{0} x, t\right)$ for $x$ in a neighborhood of $K^{i}, t \in[0, \epsilon]$.

Assume $k^{i-1}$ has been defined and let $\Delta$ be an $i$-simplex of $K^{i}, i \geqq 0$. We may assume $F_{0}$ is the identity, so $k^{i-1} F$ is the identity on a neighborhood of $\partial \Delta$. Let $\Delta \times E^{q-i}$ be contained in a neighborhood of $\Delta$; from the arguments in [5] we obtain an isotopy: $\Delta \times E^{q-i} \times[0, \epsilon]$ $\rightarrow \Delta \times E^{q-i} \times[0, \epsilon]$ which agrees with $k^{i-1} F$ on a neighborhood of $\left[\Delta \times 0 \cup \partial \Delta \times E^{q-i}\right] \times[0, \epsilon]$.

\footnotetext{
Robert Edwards has proved this result independently.
} 
The Sublemma then provides homeomorphisms $g_{t}^{i}$ with $g_{t}^{i}=k_{t}^{2-1} F_{t}$ on a neighborhood of $\Delta \times 0 \cup \partial \Delta \times E^{q-i}$ and fixed outside $\Delta \times E^{q-i}$. Putting together the isotopies so obtained from each of the $i$-simplexes of $K^{i}$ in turn we obtain the inverse of the required isotopy $k^{i}$. This completes the inductive step and the proof of the lemma.

We now prove a covering homotopy property for spaces of immersions. Let $A^{\prime} \subset A$ be compact subsets of $E^{q}$. To simplify notation in what follows, we write $\operatorname{Im}(A, Q)$ and $R(T A, T Q)$ for $\operatorname{Im}_{B^{q}}(A, Q)$ and $R\left(T E^{q} \mid A, T Q\right)$ respectively. Note that if $F: \Delta \times U \rightarrow \Delta \times Q$ or $F: \Delta$ $\times T U \rightarrow \Delta \times T Q$ is a simplex of $\operatorname{Im}(A, Q)$ or $R(T A, T Q), U$ a neighborhood of $A$, then the restriction $\rho F$ of $F$ to $\Delta \times U^{\prime}$ or $\Delta \times T U^{\prime}$ is a simplex of $\operatorname{Im}\left(A^{\prime}, Q\right)$ or $R\left(T A^{\prime}, T Q\right), U^{\prime}$ a neighborhood of $A^{\prime}$.

THEOREM 1. The restriction map $\operatorname{Im}(A, Q) \rightarrow \operatorname{Im}\left(A^{\prime}, Q\right)$ is a fibration, if $A=D^{k} \times D^{n-k}, A^{\prime}=\partial D^{k} \times D^{n-k}, k<\operatorname{dim} Q$.

This means the following: Let $F: I \times I^{n} \times U^{\prime} \rightarrow I \times I^{n} \times Q$ and $F_{0}^{\prime}: I^{n} \times U \rightarrow I^{n} \times Q$ be level preserving immersions, with $F(0, t, u)$ $=F_{0}^{\prime}(t, u)$. Then there is a level preserving immersion $F^{\prime}: I \times I^{n} \times U$ $\rightarrow I \times I_{x}^{n} Q$ with $F^{\prime}(0, t, u)=F_{0}^{\prime}(t, u)$ such that $F^{\prime}=F$ on $I \times I^{n} \times U^{\prime \prime}$, $U^{\prime \prime}$ a neighborhood of $A^{\prime}$ contained in $U^{\prime}$.

Proof of Theorem 1. Let $U_{0}^{\prime}$ be a neighborhood of $A^{\prime}$ in $E^{a}$ whose closure is compact and contained in $U^{\prime}$. Since $F$ is an immersion we can find $\epsilon>0$ and a level preserving embedding $p:[0, \epsilon] \times I^{n} \times U_{0}^{\prime}$ $\rightarrow[0, \epsilon] \times I^{n} \times U^{\prime}$ so that $F_{0} p_{t}=F_{t}$ for $0 \leqq t \leqq \epsilon$, where we write $F\left(t, t^{\prime}, u\right)=\left(t, F_{t}\left(t^{\prime}, u\right)\right)$.

Applying this result in either direction at any level $t$ we obtain $U_{t}^{\prime}, I_{t}=[t-\epsilon(t), t+\epsilon(t)]$ and $p^{t}$ such that $U_{t}^{\prime} \subset U^{\prime}, p^{t}: I_{t} \times I^{n} \times U_{t}^{\prime}$ $\rightarrow I_{t} \times I_{x}^{n} U^{\prime}$ is an isotopy and $F_{t} p_{s}^{t}=F_{s}, s \in I_{t}$.

By the $n$-Isotopy Theorem there is a neighborhood $U_{i}^{\prime \prime}$ of $A^{\prime}$ contained in $U_{i-1}^{\prime}$ and an isotopy $H^{t}: I_{t} \times I^{n} \times U_{i-1}^{\prime} \rightarrow I_{t} \times I^{n} \times U_{i-1}^{\prime}$ fixed outside a compact set with $H_{s}^{t} p_{t}^{t}\left(t^{\prime}, u\right)=p_{s}^{i}\left(t^{\prime}, u\right), u \in U_{t}^{\prime \prime}, U_{i-1}^{\prime}$ prescribed. As in [2], if $k<\operatorname{dim} Q$ we may reduce to the case in which this compact set lies inside $p_{s_{i-1}}^{t_{i}}\left(I^{n} \times U_{i-1}^{\prime}\right)$.

Since $I$ is compact we can write $0=t_{0}<s_{0}<t_{1}<s_{1}<\cdots<s_{k-1}<t_{k}$ $=1$ so that $\left[s_{i-1}, s_{i}\right] \subset\left(t_{i}-\epsilon\left(t_{i}\right), t_{i}+\epsilon\left(t_{i}\right)\right)$ for all $i$. Suppose inductively that a level presel ving immersion $F^{\prime}:\left[0, s_{i-1}\right] \times I^{n} \times U \rightarrow\left[0, s_{i-1}\right]$ $\times I^{n} \times Q$ has been defined with $F_{t}^{\prime}\left(t^{\prime}, u\right)=F_{t}\left(t^{\prime}, u\right)$ for $u \in U_{t-1}^{\prime}$, a neighborhood of $A^{\prime}$ in $U^{\prime}$.

Extend $F^{\prime}$ over $\left[0, s_{i}\right] \times I^{n} \times U$ as follows.

$$
\begin{aligned}
& F_{t}^{\prime}\left(t^{\prime}, u\right)=F_{t_{i}} H_{t}^{t_{i}}\left(B_{s_{i-1}}^{t_{i}}\right)^{-1} P_{s_{i-1}}^{t_{i}}\left(t^{\prime}, u\right), \quad u \in U_{i-1}^{\prime}, \\
& F_{t}^{\prime}\left(t^{\prime}, u\right)=F_{s_{i-1}}^{\prime}\left(t^{\prime}, u\right), \quad u \in U-U_{i-1}^{\prime}, s_{i-1} \leqq t \leqq s_{i} .
\end{aligned}
$$


Note that for $u$ in a neighborhood of $\partial U^{\prime}$ in $\bar{U}^{\prime}, H_{i}^{t_{i}}=1$ so that this extension is a well-defined immersion. A calculation shows that if $u \in U_{i}^{\prime}=U_{i-1}^{\prime} \cap U_{u}^{\prime \prime}, F_{t}^{\prime}\left(t^{\prime}, u\right)=F_{t}\left(t^{\prime}, u\right)$. This completes the inductive step; Theorem 1 is proved by starting the induction with $F_{0}^{\prime}$.

Let $D^{k} \times D^{n-k} \subset E^{a}$; we identify $\partial D^{k} \times D^{n-k+1}$ with a neighborhood of $\partial D^{k} \times D^{n-k}$ in $D^{k} \times D^{n-k}$. Let $U, U^{\prime}$ be neighborhoods of $D^{k} \times D^{n-k}$ and $\partial D^{k} \times D^{n-k+1}$ respectively in $E^{q}$, and let $\phi$ be the $U^{\prime}$ germ of an immersion $f$ of $U$ in $Q$.

Write $\operatorname{Im}_{\phi}\left(D^{k} \times D^{n-k}, Q\right)$ for the semisimplicial complex of $E^{q}$ immersions of $D^{k} \times D^{n-k}$ in $Q$ whose $\partial D^{k} \times D^{n-k+1}$ germ is equal to $\phi$. Similarly, let $R_{\phi}\left(T\left(D^{k} \times D^{n-k}\right), T Q\right)$ be the semisimplicial complex of representation germs of $T U$ in $T Q$ whose restriction to $U^{\prime}$ is $d \phi$.

Now by an argument formally identical to that in [2], Theorem 1 implies

LeMma 1. The map $d: \operatorname{Im}_{\phi}\left(D^{k} \times D^{n-k}, Q\right) \rightarrow R_{\phi}\left(T\left(D^{k} \times D^{n-k}\right), T Q\right)$ is a homotopy equivalence if $k<\operatorname{dim} Q$.

LEMMA 2. The map $d: \operatorname{Im}\left(\partial D^{k+1} \times D^{n-k}, Q\right) \rightarrow R\left(T\left(\partial D^{k+1} \times D^{n-k}\right), T Q\right)$ is a homotopy equivalence if $k<\operatorname{dim} Q$.

Now let $M, M^{\prime}$ and $Q$ be topological manifolds, $M$ a compact locally flat submanifold of the open manifold $M^{\prime}$, with $\operatorname{dim} M^{\prime}$ $=\operatorname{dim} Q$. Suppose $M$ has a handlebody decomposition with all handles of index $<\operatorname{dim} Q$.

THE IMMERSION THEOREM. The map $d: \operatorname{Im}_{M^{\prime}}(M, Q) \rightarrow R\left(T M^{\prime} \mid M, T Q\right)$ is a homotopy equivalence.

Proof. We argue by induction on the number of handles of $M$. Suppose $M=M_{0} \cup D^{k} \times D^{n-k}, M_{0} \cap D^{k} \times D^{n-k}=\partial D^{k} \times D^{n-k+1}$. Since $M$ is locally flat in $M^{\prime}, D^{k} \times D^{n-k}$ is contained in a coordinate neighborhood in $M^{\prime}$; thus by Theorem 1 , the map $\operatorname{Im}_{M^{\prime}}(M, Q) \rightarrow \operatorname{Im}_{M^{\prime}}\left(M_{0}, Q\right)$ induced by restriction, is a fibration. The proof now proceeds by an argument formally identical to that in [2].

We now use the Immersion Theorem to do surgery on topological manifolds (see [5]). Let $M^{n}$ be a topological manifold, $T M$ the tangent microbundle of $M$ and $f_{0}: S^{p} \rightarrow M$ a continuous map.

LEMMA 3. If $2 p<n$ and the induced bundle $f_{0}^{*} T M$ is trivial, there is an embedding $f: S^{p} \times D^{n-p} \rightarrow M$ which represents the homotopy class of $f_{0}$.

Proof. Let $\pi: S^{p} \times R^{n-p} \rightarrow S^{p}$ be the natural projection. Then $\left(f_{0} \pi\right)^{*} T M$ is trivial, thus the standard trivialization of $T\left(S^{p} \times R^{n-p}\right)$ induces a representation of $T R^{n} \mid S^{p}$ in $T M\left(S^{p} \times R^{n-p} C R^{n}\right)$. By the 
Immersion Theorem, there is a regular homotopy class of $E^{n}$ immersions of $S^{p}$ in $M$ corresponding to this representation. The result below shows that such a regular homotopy class contains an embedding, if $2 p<n$. The proof of Lemma 3 is completed by noting that an $E^{n}$ embedding of $S^{p}$ in $M$ restricts to an embedding of $S^{p} \times D^{n-p}$ in $M$.

Using Cernavskir's Theorem [1] and General Position arguments we can show the following

THEOREM. Let $M^{n}$ be a topological manifold and $K$ a p-complex in $E^{n}$, with $2 p<n$. Then any regular homotopy class of $E^{n}$ immersions of $K$ in $M$ contains an immersion $f: U \rightarrow M$ with $f \mid K$ an embedding.

Note that $f$ is then an embedding on a neighborhood of $K$.

THEOREM 2. Let $M^{n}$ be an almost parallelizable topological manifold. Let $\lambda \in \pi_{p} M$, with $2 p<n$. Then $\lambda$ can be represented by an embedding $f: S^{p} \times D^{n-p} \rightarrow M$ such that the manifold $\chi(M, f)$ obtained from $M$ by surgery is also almost parallelizable.

Proof of Theorem 2. Let $\Phi$ be a trivialization of $T(M-x)$. As in Lemma 3, we can find an embedding $f: S^{p} \times R^{n-p} \rightarrow M-x$ such that the trivialization of the restriction of $T M$ to $f\left(S^{p} \times R^{n-p}\right)$ induced by $d f$ from the standard trivialization of $T\left(S^{p} \times R^{n-p}\right)$ is homotopic to $\Phi \mid f\left(S^{p} \times R^{n-p}\right)$.

Now $\chi(M, f)=M-f\left(S^{p} \times D^{n-p}\right) \cup D^{p+1} \times S^{n-p-1}$ where $\left(\theta, \theta^{\prime}\right) \in S^{p}$ $\times S^{n-p-1}$ is identified with $f\left(\theta, \theta^{\prime}\right)$. Let

$$
\chi^{\prime}=S^{p} \times\left(R^{n-p}-\stackrel{\circ}{D}^{n-p}\right) \cup D^{p+1} \times 1 \times \stackrel{\circ}{D}^{n-p-1},
$$

where $1 \times D^{n-p-1} C \partial\left(D^{1} \times D^{n-p-1}\right)=S^{n-p-1}$. Now the standard trivialization of $T\left(S^{p} \times R^{n-p}\right)$ restricted to $S^{p} \times\left(R^{n-p}-\check{D}^{n-p}\right)$ extends to a trivialization of $T \chi^{\prime}$, identifying $\left(S^{p} \times 1\right) \times D^{n-p-1} C S^{p} \times D^{1} \times D^{n-p-1}$ $\subset R^{p+1} \times R^{n-p-1}$ with $\partial D^{p+1} \times\left(1 \times \dot{D}^{n-p-1}\right)$.

By the Covering Homotopy Theorem [8], this implies $\Phi \mid M$ $-f\left(S^{p} \times \dot{D}^{n-p}\right)$ extends to a trivialization of $T\left(M-f\left(S^{p} \times \dot{D}^{n-p}\right) \cup \chi^{\prime}\right)$, where $\left(\theta, t \theta^{\prime}\right) \in S^{p} \times\left(R^{n-p}-D^{n-p}\right) \subset \chi^{\prime}$ is identified with $f\left(\theta, t \theta^{\prime}\right), t \geqq 1$. However, $M-f\left(S^{p} \times \dot{D}^{n-p}\right) \cup \chi^{\prime}=\chi(M, f)-D^{n} \approx \chi(M, f)-y, y \in \dot{D}^{n}$. Thus $T(\chi(M, f)-x \cup y)$ is trivial. We may suppose $x \cup y$ is contained in a coordinate neighborhood $\psi R^{n}$, thus $T(\chi(M, f)-\psi(0))$ is trivial.

Using the surgery techniques of [3] we have applied Theorem 2 together with the Immersion Theorem to show the following

TheOREM 3. Let $M^{4 k+1}$ be a closed almost parallelizable topological manifold, which has the homotopy type of a finite complex. Then $M$ is triangulable as a piecewise linear manifold. 
R. Lashof has generalized this result in [6], thus we omit the proof.

\section{BIBLIOGRAPHY}

1. A. V. Cernavskir, On topological imbeddings of polyhedrons into Euclidean spaces, Dokl. Acad. Nauk SSSR 165 (1965), 1257-1260=Soviet Math. Dokl. 6 (1965), 1606-1610.

2. A. Haefliger and V. Poenaru, La classification des immersions combinatoires, Inst. Hautes Etudes Sci. Publ. Math. 23 (1964), 75-91.

3. M. Kervaire and J. Milnor, Groups of homotopy spheres. I, Ann. of Math. (2) 77 (1963), 504-537.

4. R. Kirby, Stable homeomorphisms, Mimeographed Notes, Institute for Advanced Study, Princeton, N. J., 1968.

5. J. M. Kister, Microbundles are fibre bundles, Ann. of Math. (2) 80 (1964), 190-199.

6. R. Lashof, Lees' immersion theorem and the triangulation of manifolds, Bull. Amer. Math. Soc. 75 (1969), 535-538.

7. J. Milnor, A procedure for killing homotopy groups of differentiable manifolds, Proc. Sympos. Pure Math., vol. 3, Amer. Math. Soc., Providence, R. I., 1961, pp. 39-55.

8. —, Microbundles. I, Topology 3 (1964), 53-80.

Rice University, Houston, Texas 77001 